\title{
MASS TREATMENT TO ELIMINATE FILARIASIS IN PAPUA NEW GUINEA
}

\author{
Moses J. Bockarie, Ph.D., Daniel J. Tisch, M.P.H., Will Kastens, B.Sc., Neal D.E. Alexander, Ph.D., \\ Zachary Dimber, Florence Bockarie, Ervin Ibam, Michael P. Alpers, M.D., and James W. Kazura, M.D.
}

\section{ABStRACT}

Background The global initiative to eradicate bancroftian filariasis currently relies on mass treatment with four to six annual doses of antifilarial drugs. The goal is to reduce the reservoir of microfilariae in the blood to a level that is insufficient to maintain transmission by the mosquito vector.

Methods In nearly 2500 residents of Papua New Guinea, we prospectively assessed the effects of four annual treatments with a single dose of diethylcarbamazine plus ivermectin or diethylcarbamazine alone on the incidence of microfilariae-positive infections, the severity of lymphatic disease, and the rate of transmission of Wuchereria bancrofti by mosquitoes. Random assignment to treatment regimens was carried out according to the village of residence, and villages were categorized as having moderate or high rates of transmission.

Results The four annual treatments with either drug regimen were taken by 77 to 86 percent of the members of the population who were at least five years old; treatments were well tolerated. The proportion with microfilariae-positive infections decreased by 86 to 98 percent, with a greater reduction in areas with a moderate rate of transmission than in those with a high rate. The respective aggregate frequencies of hydrocele and leg lymphedema were 15 percent and 5 percent before the trial began, and 5 percent $(P<0.001)$ and 4 percent $(P=0.04)$ after five years. Hydrocele and leg lymphedema were eliminated in 87 percent and 69 percent, respectively, of those who had these conditions at the outset. The rate of transmission by mosquitoes decreased substantially, and new microfilariae-positive infections in children were almost completely prevented over the five-year study period.

Conclusions Annual mass treatment with drugs such as diethylcarbamazine can virtually eliminate the reservoir of microfilariae and greatly reduce the frequency of clinical lymphatic abnormalities due to bancroftian filariasis. Eradication may be possible in areas with moderate rates of transmission, but longer periods of treatment or additional control measures may be necessary in areas with high rates of transmission. (N Engl J Med 2002;347:1841-8.)

Copyright $\odot 2002$ Massachusetts Medical Society.
B ANCROFTIAN filariasis causes elephantiasis, hydrocele, and economic loss throughout the tropics and subtropics. A global plan for its eradication is based on the notion that mass treatment with antifilarial drugs will reduce the reservoir of microfilariae in the blood to a level that is insufficient to sustain transmission by the mosquito vector. $^{1-3}$ The feasibility of the strategy is supported by observations that drugs given only once per year (diethylcarbamazine alone, diethylcarbamazine plus ivermectin, albendazole plus ivermectin, or albendazole plus diethylcarbamazine) decrease the reservoir of microfilariae. ${ }^{4-6}$ The threshold below which transmission will be interrupted is not known, but mathematical models that assume the reproductive life span of Wuchereria bancroft $i$ is approximately five years predict that annual mass treatment for four to six years will be required.7,8

In 1994 we initiated a trial comparing the efficacy of diethylcarbamazine plus ivermectin with that of diethylcarbamazine alone in limiting the transmission of filariasis in Papua New Guinea. The proportion of persons who were positive for microfilariae decreased by 76 to 99 percent, and the transmission potential the estimated number of infective (third-stage) larvae inoculated per person per year - decreased by 31 to 58 percent 12 months after a single dose of the drugs. ${ }^{9}$ In the current study, we assessed the influence of baseline transmission levels and the effect of four annual antifilarial treatments on three key end points of filariasis control: the reservoir of microfilariae, the severity of lymphatic abnormalities, and the incidence of new infections.

\section{METHODS}

\section{Study Population and Randomization}

Residents of 14 villages (hereafter referred to as units or treatment units) in the Dreikikir district of Papua New Guinea participated in this open-label study comparing the efficacy of a single annual dose of diethylcarbamazine plus ivermectin $(6 \mathrm{mg}$ per kilogram of body

From the Papua New Guinea Institute of Medical Research, Goroka, Madang, and Maprik, Papua New Guinea (M.J.B., W.K., Z.D., F.B., E.I., M.P.A.); the Division of Geographic Medicine and Center for International Health, Case Western Reserve University School of Medicine and University Hospitals of Cleveland, Cleveland (D.J.T., W.K., J.W.K.); the London School of Hygiene and Tropical Medicine, London (N.D.E.A.); and the Center for International Health, Curtin University, Perth, Australia (M.P.A.). Address reprint requests to Dr. Kazura at the Division of Geographic Medicine and Center for International Health, Case Western Reserve University School of Medicine and University Hospitals of Cleveland, 2109 Adelbert Rd., W137 Harlan Wood Bldg., Cleveland, OH 44106, or at jxk14@po.cwru.edu. 
weight and $400 \mu \mathrm{g}$ per kilogram, respectively) with that of a single annual dose of diethylcarbamazine alone (6 mg per kilogram) in reducing the transmission of $W$. bancrofti. The method of randomization and the categorization of 8 treatment units as having a moderate rate of transmission and of 6 as having a high rate of transmission have been described previously. . $^{-12}$

The procedure for oral informed consent and the study design were approved by the Medical Research Advisory Committee of Papua New Guinea and the institutional review board for human investigations at Case Western Reserve University in Cleveland. Consent for the participation of subjects who were 16 years old or younger was obtained from a parent or guardian. Only persons who were at least five years old were eligible to receive drugs. Children who reached their fifth birthday within the 12 -month period preceding an annual treatment were given their first treatment at that time. Pregnant women were excluded from receiving the drugs, because ivermectin cannot be used during pregnancy.

\section{Drug Distribution and Adverse Effects}

Diethylcarbamazine (50-mg tablets) and ivermectin (1-mg tablets) were supplied by the World Health Organization. Because reactions to antifilarial drugs are most severe in persons with high levels of microfilariae, ${ }^{13,14}$ we closely monitored 966 subjects for side effects after the first treatment. Minor reactions (mvalgia, headache, fatigue, and swelling of an arm, a leg, or genitalia) and severe reactions (high fever, shortness of breath, and collapse) that occurred within 48 hours after drug administration were recorded. Acetaminophen was given for minor reactions.

\section{Measures of Infection and Disease}

Infection status was evaluated one day before the first treatment in 1994 and yearly after the first, second, third, and fourth annual treatments. The density of microfilariae in $1 \mathrm{ml}$ of blood and the $\mathrm{Og} 4 \mathrm{C} 3$ antigen level were quantified by membrane filtration and enzyme-linked immunosorbent assay (TropBioMed), respectively. ${ }^{15,16}$ The proportion of subjects who were positive for microfilariae and the geometric mean number of microfilariae per milliliter of blood before and one year after each treatment were calculated.

Physical examination was performed annually, and the severity of lymphatic abnormalities was graded according to standard criteria. ${ }^{17}$ Only subjects who were at risk for advanced hydrocele (male subjects who were at least 16 years old) and moderate-to-severe lymphedema of the legs (subjects who were at least 21 years old) were scored as positive for disease. An adult with lymphedema of the legs and arms is shown in Figure 1.

\section{Entomologic Studies}

Mosquitoes were captured for four nights per month as they landed on the legs of adult residents of the study area. The mosquito species was determined, and insects were examined for infective (third-stage) larvae. ${ }^{18,19}$ The biting rate (the number of mosquitoes attempting to take a blood meal per person), the infective biting rate (the number of Anopheles punctulatus that had at least one infective larva), and the transmission potential were determined. ${ }^{20,21}$

\section{Statistical Analysis}

The frequency of adverse reactions according to the treatment regimen, differences in the proportion of subjects who were positive for microfilariae before and after each treatment, and changes in the antigen level and the severity of lymphatic abnormalities were evaluated for significance with the use of chi-square tests. Differences in the rates of transmission before and after treatment were evaluated with use of the Mann-Whitney U test. A generalized estimating equation was used to assess the difference in the odds of having a microfilariae-positive infection according to the treatment regimen and rate of transmission (moderate or high) after adjustment for the correlation of observations within treatment units over time. ${ }^{22}$ The

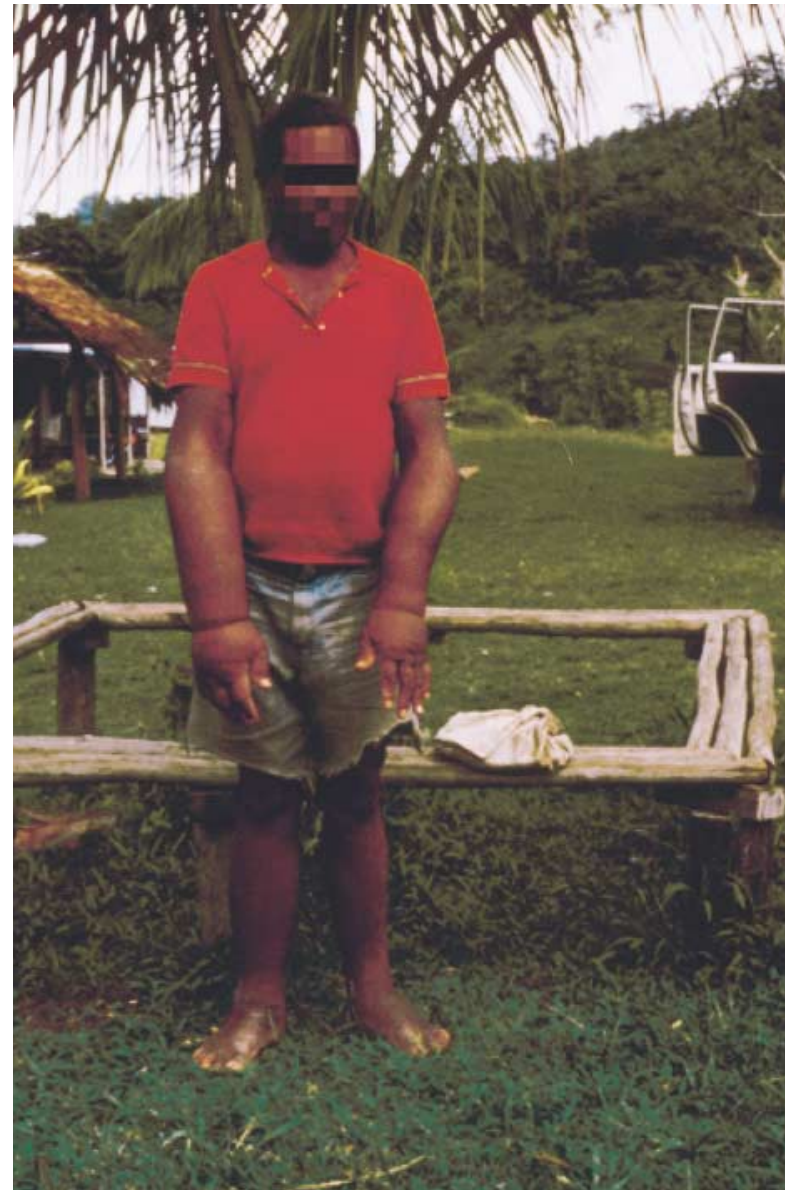

Figure 1. A 52-Year-Old Papua New Guinea Man with Elephantiasis of the Legs and Lymphedema of the Arms.

This man also had an advanced hydrocele.

logarithmic odds of lymphedema of the legs and hydrocele were modeled with use of the same technique. The resulting estimates were converted to odds ratios with 95 percent confidence intervals calculated from the robust estimator of variance. Modeling was performed with SAS 8.2 software (SAS Institute) with use of the GENMOD procedure.

\section{RESULTS}

\section{Community Participation in Mass Treatment}

A total of 2586 persons were eligible to take antifilarial drugs in 1994, 2668 were eligible in 1995, 2695 were eligible in 1996, and 2690 were eligible in 1997. Ineligible persons ( 14 percent of the de facto population) were primarily children younger than five years old. Eighty-six percent of eligible subjects took antifilarial drugs during the first annual treatment, 78 percent during the second, 80 percent during the third, and 77 percent during the fourth. Of the 2586 subjects enrolled in 1994, 1 percent received no doses of 
antifilarial drugs, 19 percent received one dose, 21 percent received two doses, 24 percent received three doses, and 35 percent received all four doses. Fifty percent of the episodes of noncompliance were not random (e.g., half the subjects who did not take the medication in 1997 also did not do so in 1995 and 1996). There were no significant differences between the number of doses of diethylcarbamazine plus ivermectin that were taken and the number of doses of diethylcarbamazine alone that were taken.

\section{Adverse Reactions}

No severe reactions were observed. Minor reactions were reported after the first treatment by 20 percent of subjects who took diethylcarbamazine plus ivermectin (93 of 472) and 11 percent of subjects who took diethylcarbamazine alone $(53$ of 494$)\left(\chi^{2}=14.3, \mathrm{P}<\right.$ $0.01)$. Seven hundred fifty-four of these subjects took the medications the following year. Nine of 146 subjects who had minor side effects after the first treatment ( 6 percent) declined to take the second annual dose, as compared with 111 of 608 who did not have an adverse reaction after the first treatment (18 percent $)\left(\chi^{2}=11.9, \mathrm{P}<0.01\right)$.

\section{Effect of Treatment on the Reservoir of Microfilariae}

Annual treatment reduced the reservoir of microfilariae (Table 1). The initial decrease was greatest in treatment units with a moderate rate of transmission whose inhabitants were randomly assigned to receive diethylcarbamazine plus ivermectin. Forty-seven percent of such subjects were positive for microfilariae before the initial treatment, as compared with 1 percent one year after the fourth treatment $\left(\chi^{2}=475.9\right.$, $\mathrm{P}<0.001)$. In units with a high rate of transmission, the rate of positivity for microfilariae decreased from 77 percent to 5 percent during the same period $\left(\chi^{2}=\right.$ $287.5, \mathrm{P}<0.001)$. In units whose inhabitants were randomly assigned to receive diethylcarbamazine alone, the rates decreased from 42 percent before the initial treatment to 2 percent one year after the fourth treatment in areas with a moderate rate of transmission and from 76 percent to 11 percent in areas with a high rate of transmission.

A generalized estimating equation was used to account for the correlation of observations clustered within various treatment units. The model to estimate the odds of microfilarial infection included the drug regimen, the estimated transmission potential before treatment, the year of observation, and an interaction term for year and treatment regimen that was based on a logit link and an independent working correlation structure. The efficacy of diethylcarbamazine plus ivermectin was greater than that of diethylcarbamazine alone one year after the first, second, and third annual treatments (Fig. 2). For example, after the third treatment, residents of sites randomly assigned to receive diethylcarbamazine alone were 3.3 times as likely to be positive for microfilariae as those who lived in areas where diethylcarbamazine plus ivermectin was given (95 percent confidence interval, 1.04 to $10.57 ; \mathrm{P}=$ $0.04)$, and the odds of being positive for microfilariae were 3.5 times as great for residents of areas with a high rate of transmission as for residents of areas

Table 1. Effect of Four Annual Treatments with Diethylcarbamazine plus Ivermectin or Diethylcarbamazine Alone on the Reservoir of Microfilariae in Treatment Units with a Moderate Rate of Transmission and Treatment Units with a High Rate of Transmission, According to Year.*

Treatment AND Variable

Diethylcarbamazine plus ivermectin

No. of subjects examined

No. of microfilariae-positive subjects (\%)

Geometric mean no. of microfilariae $/ \mathrm{ml}$

Diethylcarbamazine alone

No. of subjects examined

No. of microfilariae-positive subjects (\%)

Geometric mean no. of microfilariae $/ \mathrm{ml}$

\begin{tabular}{lcccc}
\multicolumn{5}{c}{ Moderate transmission Rate } \\
\hline 1994 & 1995 & 1996 & 1997 & 1998
\end{tabular}

\begin{tabular}{lllll}
\multicolumn{6}{c}{ High Transmission Rate } \\
\hline 1994 & 1995 & 1996 & 1997 & 1998
\end{tabular}

$\begin{array}{llccc}797 & 756 & 790 & 819 & 750 \\ 376(47) \dagger & 156(21) & 45(6) & 8(1) & 7(1) \\ & & & & \\ 9.6 & 0.9 & 0.3 & 0.3 & 0.5\end{array}$

$\begin{array}{lllll}281 & 318 & 311 & 303 & 266 \\ 216(77) \dagger & 101(32) & 74(24) & 33(11) & 14(5)\end{array}$

$\begin{array}{lllll}49.8 & 2.9 & 2.6 & 0.6 & 0.3\end{array}$

$\begin{array}{lllcc}903 & 815 & 802 & 692 & 639 \\ 381(42) \dagger & 238(29) & 121(15) & 47(7) & 10(2) \\ 8.8 & 2.6 & 0.9 & 0.3 & 0.5\end{array}$

$\begin{array}{lrlrr}243 & 192 & 253 & 257 & 165\end{array}$

$185(76) \dagger \quad 98(51) \quad 107(42) \quad 56(22) \quad 18(11)$

$\begin{array}{lllll}55.2 & 13.1 & 7.8 & 1.4 & 0.5\end{array}$

${ }^{*}$ Four treatment units with a moderate transmission rate were randomly assigned to receive diethylcarbamazine plus ivermectin, and four were assigned to receive diethylcarbamazine alone; three treatment units with a high transmission rate were randomly assigned to receive diethylcarbamazine plus ivermectin, and three were assigned to receive diethylcarbamazine alone.

$\dagger \mathrm{P}<0.001$ for the comparison with all other years. 


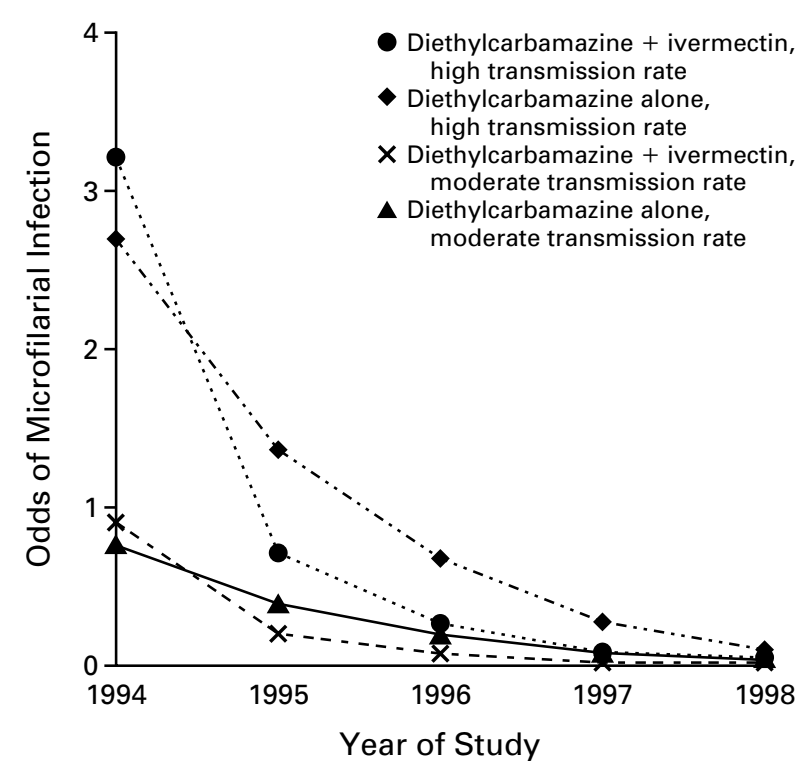

Figure 2. Odds of Microfilariae-Positive Infection over the FiveYear Study Period among Subjects in Treatment Areas with Moderate and High Rates of Transmission Randomly Assigned to Receive Diethylcarbamazine plus Ivermectin or Diethylcarbamazine Alone.

The first annual dose of each drug regimen was given immediately after the determination of microfilarial status in 1994. The odds of microfilariae-positive infection were less than 0.1 in 1998, regardless of the drug regimen or the base-line transmission potential.

with a moderate rate of transmission ( 95 percent confidence interval, 1.30 to $9.09 ; \mathrm{P}=0.02)$. After the fourth treatment, however, the odds of microfilariaepositive infection decreased to less than 0.1 , regardless of whether subjects lived in an area with a moderate or a high rate of transmission and regardless of whether they were given diethylcarbamazine plus ivermectin or diethylcarbamazine alone.

\section{Effect of Treatment on Transmission}

Before treatment, there were 24 to 167 bites from mosquitoes containing infective larvae per person per year in treatment units with a moderate rate of transmission and 224 to 742 infective bites per person per year in units with a high rate of transmission; the respective ranges for the transmission potential were 45 to 404 and 704 to 2518 infective larvae inoculated per person per year. Detailed entomologic indexes in two treatment units where diethylcarbamazine plus ivermectin was used are presented in Figure 3.

In the unit with a moderate rate of transmission, the overall transmission potential decreased by 97 percent (from 704 to 23 infective larvae inoculated per person per year). The effect of treatment was less marked in the unit with a higher rate of transmission. The pretreatment transmission potential of 1485 infective larvae inoculated per person per year decreased to 234 infective larvae inoculated per person per year the year after the third annual treatment (84 percent reduction). The pattern of exposure to infective mosquitoes also differed according to the pretreatment rate of transmission. In units with a moderate rate of transmission before treatment, after treatment there was an average of 0 bites from infective mosquitoes per person per month during 11 months of the year. In contrast, in units with a high rate of transmission before treatment, rates remained moderate to high ( 1 to more than 30 bites from infective mosquitoes per person per year) for five to seven months per year after treatment was initiated.

To estimate the effect of mass treatment on the establishment of new infections, we compared the prevalence of microfilariae-positive infections in previously untreated cohorts of five-to-six-year-old children from each year of the study for the treatment regimens combined. Before the initial treatment, 28 of 64 children (44 percent) were positive for microfilariae. A total of 9 percent were positive for microfilariae in 1995 ( 3 of 32), 11 percent in 1996 (8 of 71), 2 percent in 1997 ( 1 of 42), and 6 percent in 1998 ( 3 of 52$)\left(\chi^{2}=12.9\right.$ for the comparison between the pretreatment value and the value in $1998, \mathrm{P}<0.001)$.

\section{Effect on Filarial Antigen Level}

Estimates of the levels of circulating filarial antigen alone, independent of the blood level of microfilariae, are thought to reflect the burden of pre-adult and adult worms. ${ }^{23-25}$ Antigen levels were monitored between 1994 and 1997 in residents of two treatment units (Table 2). Fewer than 20 percent of subjects who were antigen-positive before the trial began were antigen-negative one year after the third annual treatment, though mean antigen levels decreased by 79 percent. There was no significant difference in these proportions between the two drug regimens. Changes in antigen status and level did not correlate with the number of treatments a subject received. No subjects who were identified as antigen-negative before the trial began were antigen-positive in subsequent years, and in no case did the antigen level increase relative to the pretreatment value.

\section{Effect of Treatment on Hydrocele and Leg Lymphedema}

The proportion of male subjects with advanced hydrocele who were at least 16 years old was 15 percent (110 of 726) before the trial began and 5 percent $(27$ of 563) one year after the fourth treatment $\left(\chi^{2}=35.8\right.$, $\mathrm{P}<0.001)$. There was no significant difference in the frequency or odds of hydrocele between the two drug regimens, after adjustment for the clustering of treat- 


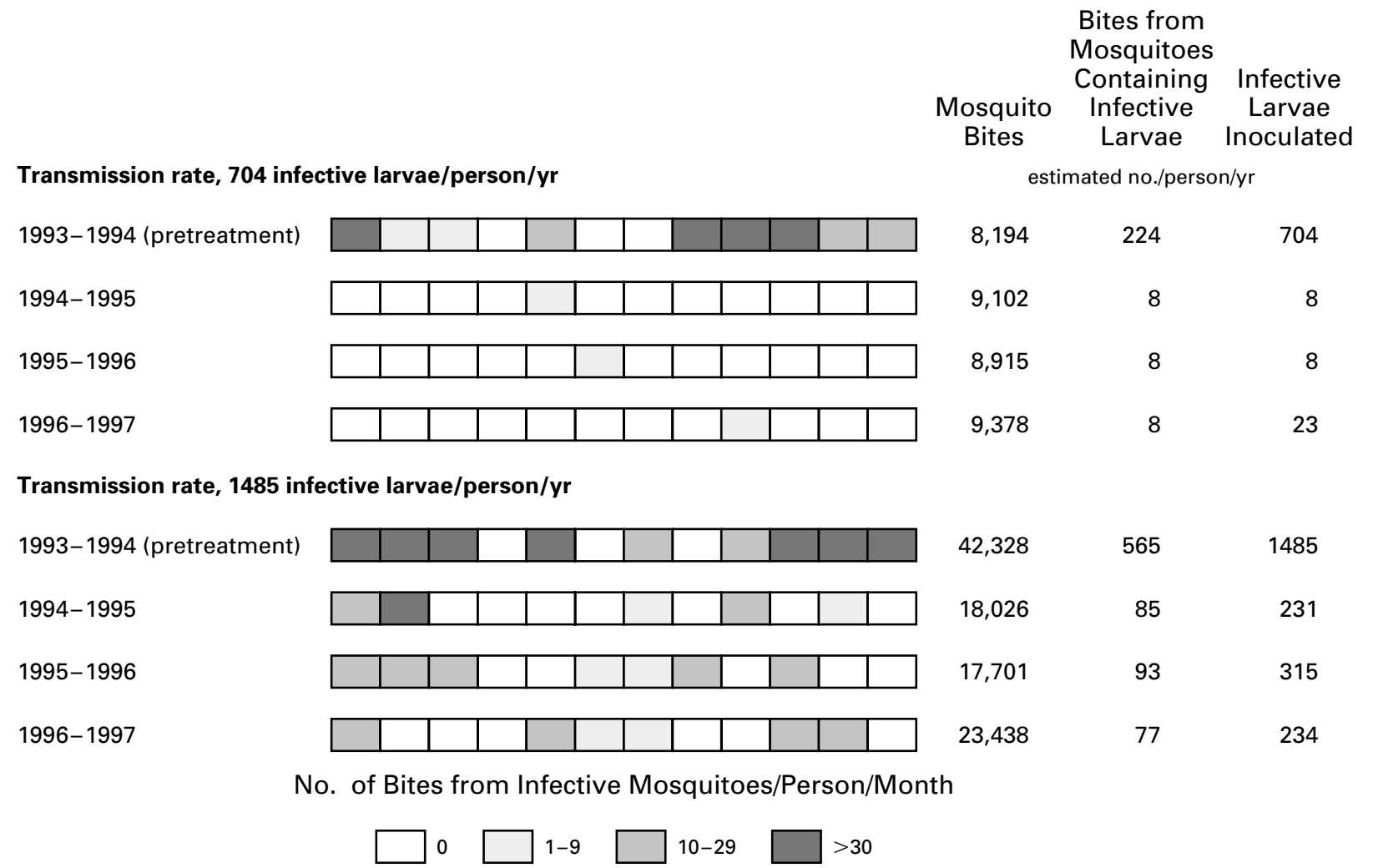

Figure 3. Entomologic Indexes of Transmission in Two Units with Differing Rates of Transmission Randomly Assigned to Receive Diethylcarbamazine plus Ivermectin.

The biting rate (the estimated number of mosquitoes attempting to take a blood meal per person per year), infective biting rate (the estimated number of bites from mosquitoes containing infective larvae per person per year), and the transmission potential (the estimated number of infective larvae inoculated per person per year) are shown. In each horizontal bar, the sections represent the 12 months of the year.

ment units and time, although the odds did decrease with each year $(\mathrm{P}=0.02)$. In addition, the odds of hydrocele were 2.4 times as great among residents of areas with a high rate of transmission as among residents of areas with a moderate rate of transmission over the four-year period of the study (95 percent confidence interval, 2.05 to $4.76 ; \mathrm{P}=0.02$ ).

The frequency of lymphedema of the legs was 5 percent before treatment ( 68 of 1273 subjects) and 4 percent after the fourth treatment ( 35 of 998 subjects; $\left.\chi^{2}=4.4 ; \mathrm{P}=0.04\right)$. There was no significant difference between the two drug regimens in the odds of lymphedema of the legs, although the odds decreased each year $(\mathrm{P}=0.03)$. Residents of units with a high rate of transmission were 3.3 times as likely to have lymphedema as residents of units with a moderate rate of transmission over the four-year study period ( 95 percent confidence interval, 1.33 to $8.33 ; \mathrm{P}=0.01$ ).

A separate analysis was performed that included subjects who had lymphatic disease at the beginning of the trial and who were monitored during the fiveyear study period. Ninety-one of 105 men who had advanced hydrocele ( 87 percent) and 62 of 90 adults who had lymphedema of the legs (69 percent) in 1993 to 1994 no longer had clinical signs of disease in 1998. The likelihood of the amelioration of lymphatic abnormalities did not correlate with the drug regimen, the number of treatments actually received, or the change in infection status as judged by the number of microfilariae or the antigen level.

\section{DISCUSSION}

In an effort to eradicate bancroftian filariasis, mass treatment with four to six annual doses of antifilarial drugs is being implemented in some parts of the world. ${ }^{26,27}$ Our findings support this strategy for areas with low-to-moderate rates of transmission in regions where anopheline mosquitoes transmit this infectious disease, such as in Melanesia and Africa. Additional measures or longer periods of treatment may be nec- 


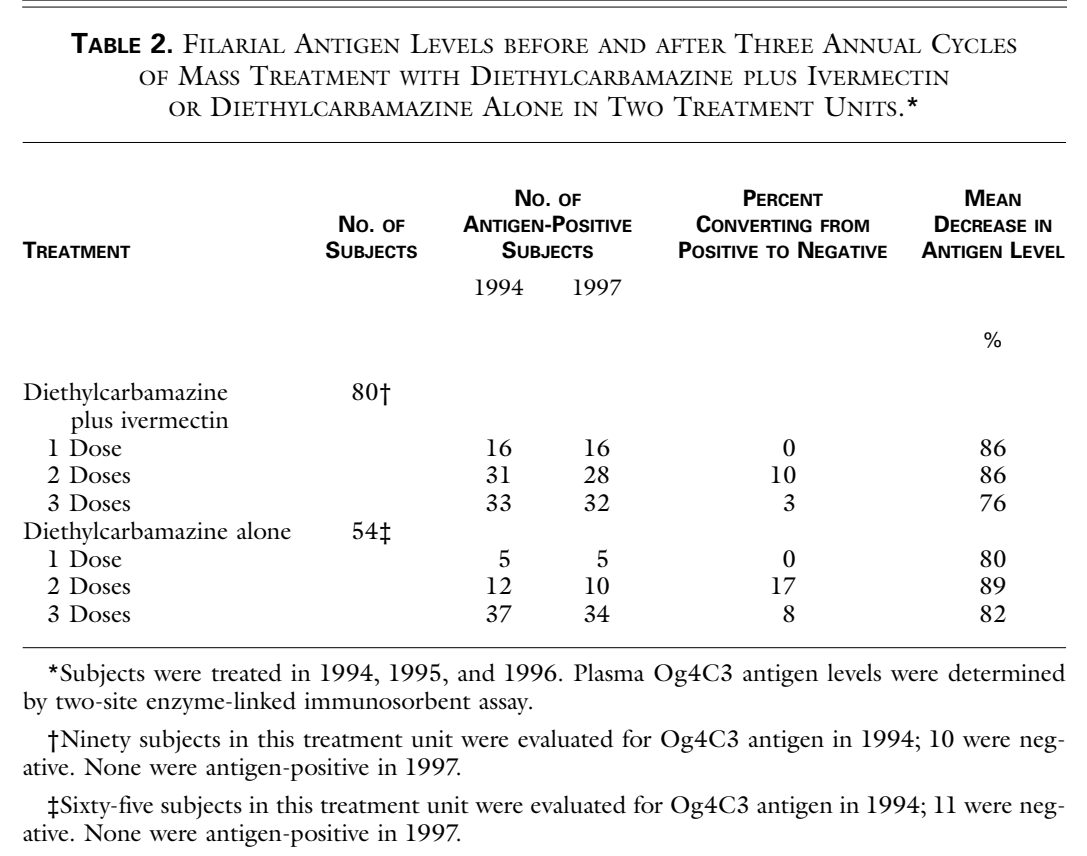

essary in areas with a high rate of transmission. Since the efficiency of transmission varies among mosquito species, it is not yet clear whether similar conclusions can be drawn in areas where non-anopheline mosquitoes transmit $W$. bancrofti.

Our results suggest that mass treatment will be well accepted. Serious adverse reactions were not observed after 8335 doses of diethylcarbamazine plus ivermectin or diethylcarbamazine alone had been given, and 77 to 86 percent of eligible subjects received at least one of the four annual treatments. Although minor side effects such as myalgia and fatigue were noted by one sixth of subjects after the first treatment, such reactions were associated with increased rates of participation the following year, possibly because adverse effects were perceived as indicators of the efficacy of the treatment. Acute post-treatment reactions may be mediated by inflammatory cytokines that are up-regulated when filarial antigens and wolbachia-derived molecules are released by dying microfilariae. 28,29

Mass treatment reduced the burden of clinical lymphatic disease. The prevalence of hydrocele decreased from 15 percent to 5 percent, and scrotal swelling disappeared in 87 percent of male subjects with preexisting genital disease. Similarly, the prevalence of lymphedema of the legs decreased from 5 percent to 4 percent, and 69 percent of subjects with preexisting disease had a clinical cure. Reports from other areas in which bancroftian filariasis is endemic suggest that the beneficial effects we observed may be generalizable. ${ }^{30,31}$ The mechanism by which overt lymphatic disease is reversed is not known, but the infection does not need to be cured, since filarial antigenemia persisted in most subjects and there was no correlation between this finding and the number of treatments actually received. We speculate that mass treatment decreases exposure to the infective larvae and that this, in turn, diminishes the intensity of inflammatory responses to newly inoculated $W$. bancrofti or their wolbachia endosymbionts. Studies have shown that the rate of transmission correlates with both the prevalence of lymphatic abnormalities and the cytokine profile and strength of filarial-specific T-cell immunity. ${ }^{32,33}$

Four annual treatments reduced the proportion of subjects who were positive for microfilariae by 86 to 98 percent and the geometric mean density of microfilariae to less than 1 in all groups. Decreases in the reservoir of microfilariae were more pronounced after treatment with diethylcarbamazine plus ivermectin than after treatment with diethylcarbamazine alone, and the reduction in the microfilariae-positive rates was initially greater in areas with a moderate rate of transmission than in areas with a high rate of transmission. After the fourth treatment, however, the odds of being positive for microfilariae were similar in all groups regardless of the rate of transmission or the drug regimen. It is important to consider these results in the context of the high rates of transmission and infection in Papua New Guinea relative to other areas of endemic infection. Microfilariae-positive rates of 20 to 30 percent and transmission potentials of approximately 400 infective larvae per person per year (defined as

1846 - N Engl J Med, Vol. 347, No. 23 • December 5, $2002 \cdot$ www.nejm.org 
a moderate rate in our study) are considered high in most regions of Africa and the Pacific islands. ${ }^{10,11,34-36}$ In addition, recommendations to include albendazole in mass-treatment programs will most likely lead to even greater efficacy, since this drug, like diethylcarbamazine, is active against adult worms.

The remarkable decreases in the reservoir of microfilariae and the rates of transmission were accompanied by an 87 percent reduction from the pretreatment value in the number of microfilariae-positive infections in previously untreated five-to-six-year-old children after the fourth annual treatment. Since the children who were evaluated after the fourth treatment were 1 to 12 months old during the pretreatment period, the few infections in this group are most likely due to exposure to infective larvae before the transmission rate had been diminished by mass treatment. If this explanation is correct, the data indicate that new infections were completely prevented after four annual treatments.

Observations from other areas in which filariasis is endemic suggest that intense courses of diethylcarbamazine given over a period of 10 or more years interrupt transmission, ${ }^{37-42}$ whereas a recent report from India, where culicine mosquitoes transmit $W$. bancrof$t i$, shows that the rates of microfilariae-positive infection decreased by 48 percent after four annual treatments with diethylcarbamazine alone. ${ }^{43}$ Comparison of these reports with our data is difficult, since none included a prospective analysis of the transmission potential, the levels of microfilariae or filarial antigen, the rate of new infections, or the influence of the baseline transmission rate.

Our results support the concept that infection and the burden of disease due to bancroftian filariasis can be reduced substantially with as few as four annual mass treatments that include single doses of diethylcarbamazine. It is not yet known whether and how long these benefits will be sustained after mass treatment ceases. Eradication may ultimately require more than four to six years of treatment, drugs with greater activity against adult worms, or adjunctive strategies that limit exposure to mosquitoes or enhance resistance to infection. Evaluation of changes in filarial ecology after the cessation of mass treatment in Papua New Guinea and other areas of endemic infection will provide this information and can be used to validate and refine mathematical models designed to predict the threshold necessary to achieve eradication. $7,8,44,45$

Supported by funds from the World Health Organization (F30/181/67) and the Papua New Guinea Institute of Medical Research. The data analysis was supported by a grant (U19 AI33061) from the Public Health Service.

\section{REFERENCES}

1. Behbehani K. Candidate parasitic diseases. Bull World Health Organ 1998;76:Suppl 2:64-7.
2. Dreyer G, Coelho G. Lymphatic filariasis: a potentially eradicable disease. Cad Saude Publica 1997;13:537-43. (In Portuguese.)

3. Ottesen EA, Ismail MM, Horton J. The role of albendazole in programmes to eliminate lymphatic filariasis. Parasitol Today 1999;15:382-6.

4. Ottesen EA. Efficacy of diethylcarbamazine in eradicating infection

with lymphatic-dwelling filariae in humans. Rev Infect Dis 1985;7:341-56.

5. Horton J, Witt C, Ottesen EA, et al. An analysis of the safety of the

single dose, two drug regimens used in programmes to eliminate lymphatic filariasis. Parasitology 2000;121:Suppl:S147-S160.

6. Addiss DG, Beach MJ, Streit TG, et al. Randomised placebo-controlled comparison of ivermectin and albendazole alone and in combination for

Wuchereria bancrofti microfilaraemia in Haitian children. Lancet 1997;

350:480-4. [Erratum, Lancet 1997;350:1036.]

7. Norman RA, Chan MS, Srividya A, et al. EPIFIL: the development of an age-structured model for describing the transmission dynamics and control of lymphatic filariasis. Epidemiol Infect 2000;124:529-41.

8. Plaisier AP, Subramanian S, Das PK, et al. The LYMFASIM simulation program for modeling lymphatic filariasis and its control. Methods Inf Med 1998;37:97-108

9. Bockarie MJ, Alexander ND, Hyun P, et al. Randomised communitybased trial of annual single-dose diethylcarbamazine with or without ivermectin against Wuchereria bancrofti infection in human beings and mosquitoes. Lancet 1998;351:162-8.

10. Bockarie M, Kazura J, Alexander N, et al. Transmission dynamics of Wuchereria bancrofti in East Sepik Province, Papua New Guinea. Am J Trop Med Hyg 1996;54:577-81.

11. Michael E, Simonsen PE, Malecela $M$, et al. Transmission intensity and the immunoepidemiology of bancroftian filariasis in East Africa. Parasite Immunol 2001;23:373-88.

12. Smith PG, Morrow RH, eds. Methods for field trials of intervention against tropical diseases: a "toolbox." Oxford, England: Oxford University Press, 1991.

13. Kazura J, Greenberg J, Perry R, Weil G, Day K, Alpers M. Comparison of single-dose diethylcarbamazine and ivermectin for treatment of bancroftian filariasis in Papua New Guinea. Am J Trop Med Hyg 1993;49:804 11.

14. Ottesen EA, Vijayasekaran V, Kumaraswami V, et al. A controlled trial of ivermectin and diethylcarbamazine in lymphatic filariasis. N Engl J Med 1990;322:1113-7.

15. Desowitz RS, Hitchcock JC. Hyperendemic bancroftian filariasis in the Kingdom of Tonga: the application of the membrane filter concentration technique to an age-stratified blood survey. Am J Trop Med Hyg $1974 ; 23: 877-9$

16. More SJ, Copeman DB. A highly specific and sensitive monoclonal antibody-based ELISA for the detection of circulating antigen in bancroftian filariasis. Trop Med Parasitol 1990;41:403-6.

17. WHO Expert Committee on Filariasis. Third report. WHO Tech Rep Ser $1974 ; 542: 1-54$

18. Bockarie MJ, Fischer P, Williams SA, et al. Application of a polymerase chain reaction-ELISA to detect Wuchereria bancrofti in pools of wildcaught Anopheles punctulatus in a filariasis control area in Papua New Guinea. Am J Trop Med Hyg 2000;62:363-7.

19. Hii JL, Smith T, Mai A, Ibam E, Alpers MP. Comparison between anopheline mosquitoes (Diptera: Culicidae) caught using different methods in a malaria endemic area of Papua New Guinea. Bull Entomol Res 2000;90:211-9.

20. Nelson GS. Staining of filarial larvae in insects before dissection. Bull World Health Organ 1958;19:204

21. Walsh JF, Davies JB, Le Berre R, Grams R. Standardization of criteria for assessing the effect of Simulium control in onchocerciasis control programmes. Trans R Soc Trop Med Hyg 1978;72:675-6.

22. Liang K-Y, Zeger SL. Longitudinal data analysis using generalized linear models. Biometrika 1986;73:13-22.

23. Weil GJ, Lammie PJ, Richards FO Jr, Eberhard ML. Changes in circulating parasite antigen levels after treatment of bancroftian filariasis with diethylcarbamazine and ivermectin. J Infect Dis 1991;164:814-6.

24. Freedman DO, Plier DA, De Almeida AB, De Oliveira AL, Miranda J, Braga C. Effect of aggressive prolonged diethylcarbamazine therapy on circulating antigen levels in bancroftian filariasis. Trop Med Int Health 2001;6:37-41

25. Tisch DJ, Hazlett FE, Kastens W, Alpers MP, Bockarie MJ, Kazura JW. Ecologic and biologic determinants of filarial antigenemia in bancroftian filariasis in Papua New Guinea. J Infect Dis 2001;184:898-904. 26. Dean $M$. At last, the fight against lymphatic filariasis begins. Lancet 2000;355:385.

27. Burkot $T$, Ichimori $K$. The PacELF programme: will mass drug administration be enough? Trends Parasitol 2002;18:109-15. 
28. Ismail MM, Jayakody RL, Weil GJ, et al. Efficacy of single dose combinations of albendazole, ivermectin and diethylcarbamazine for the treatment of bancroftian filariasis. Trans R Soc Trop Med Hyg 1998;92:94-7. 29. Taylor MJ, Bandi C, Hoerauf AM, Lazdins J. Wolbachia bacteria of filarial nematodes: a target for control? Parasitol Today 2000;16:179-80. 30. Meyrowitsch DW, Simonsen PE, Makunde WH. Mass DEC chemotherapy for control of bancroftian filariasis: comparative efficacy of four strategies two years after start of treatment. Trans R Soc Trop Med Hyg 1996;90:423-8.

31. Partono F. Treatment of elephantiasis in a community with timorian filariasis. Trans R Soc Trop Med Hyg 1985;79:44-6.

32. Kazura JW, Bockarie M, Alexander N, et al. Transmission intensity and its relationship to infection and disease due to Wuchereria bancrofti in Papua New Guinea. J Infect Dis 1997;176:242-6.

33. King CL. Transmission intensity and human immune responses to lymphatic filariasis. Parasite Immunol 2001;23:363-71.

34. Southgate BA, Bryan JH. Factors affecting transmission of Wuchereria bancrofti by anopheline mosquitoes. 4 . Facilitation, limitation, proportionality and their epidemiological significance. Trans R Soc Trop Med Hyg 1992;86:523-30

35. Webber RH. Vector control of filariasis in the Solomon Islands. Southeast Asian J Trop Med Public Health 1975;6:430-4.

36. Hawking F. The distribution of human filariasis throughout the world. II. Asia. Trop Dis Bull 1976;73:967-1016

37. Fan PC. Filariasis eradication on Kinmen Proper, Kinmen (Quemoy) Islands, Republic of China. Acta Trop 1990;47:161-9.
38. Perolat P, Guidi C, Rivière F, Roux J. Filariose de bancroft Polynésie française: situation épidémiologique et perspectives après 35 ans de lutte. Bull Soc Pathol Exot Filiales 1986;79:78-88.

39. Esterre P, Plichart C, Sechan Y, Nguyen NL. The impact of 34 years of massive DEC chemotherapy on Wuchereria bancrofti infection and transmission: the Maupiti cohort. Trop Med Int Health 2001;6:190-5. 40. Mataika JU, Kimura E, Koroivueta J, Shimada M. Efficacy of five annual single doses of diethylcarbamazine for treatment of lymphatic filariasis in Fiji. Bull World Health Organ 1998;76:575-9.

41. Maxwell CA, Curtis CF, Haji H, Kisumku S, Thalib AI, Yahya SA. Control of bancroftian filariasis by integrating therapy with vector control using polystyrene beads in wet pit latrines. Trans R Soc Trop Med Hyg 1990;84:709-14.

42. Zielke E, Chlebowsky HO. Studies on bancroftian filariasis in Liberia, West Africa. V. The influence of treatment with diethylcarbamazine and vector control on the transmission of Wuchereria bancrofti. Tropenmed Parasitol 1980;31:444-58.

43. Das PK, Ramaiah KD, Vanamail $P$, et al. Placebo-controlled community trial of four cycles of single-dose diethylcarbamazine or ivermectin against Wuchereria bancrofti infection and transmission in India. Trans R Soc Trop Med Hyg 2001;95:336-41.

44. Rochet MJ. A simple deterministic model for bancroftian filariasis transmission dynamics. Trop Med Parasitol 1990;41:225-33.

45. Srividya A, Das PK, Subramanian S, et al. Past exposure and the dynamics of lymphatic filariasis infection in young children. Epidemiol Infect 1996;117:195-201.

Copyright (C) 2002 Massachusetts Medical Society.

\section{ELECTRONIC ACCESS TO THE JOURNAL'S CUMULATIVE INDEX}

At the Journal's site on the World Wide Web (http://www.nejm.org) you can search an index of all articles published since January 1975 (abstracts 1975-1992, full-text 1993present). You can search by author, key word, title, type of article, and date. The results will include the citations for the articles plus links to the abstracts of articles published since 1993. For nonsubscribers, time-limited access to single articles and 24-hour site access can also be ordered for a fee through the Internet (http://www.nejm.org).

1848 - N Engl J Med, Vol. 347, No. 23 • December 5, $2002 \cdot$ www.nejm.org 